\title{
NOTE ON A SPECIAL CYCLIC SYSTEM*
}

\section{BY MALCOLM FOSTER}

1. Introduction. This note is concerned with a special cyclic system. $\uparrow$ Let $S$ be a surface referred to any orthogonal system, and $T$ the trihedral whose $x$-axis is tangent to the curve $v=$ const. The equations

$$
x=R(1+\cos \theta), \quad y=0, \quad z=R \sin \theta,
$$

define a two-parameter family of circles $C$ normal to $S$; and the necessary and sufficient conditions that $C$ shall constitute a cyclic system are

(2) $\xi \frac{\partial R}{\partial v}+R \eta_{1} r=0, \quad R\left(p r_{1}-p_{1} r\right)-q_{1}\left(\xi+\frac{\partial R}{\partial u}\right)+q \frac{\partial R}{\partial v}=0$.

It is readily seen that the first of equations (2) may be written $\ddagger$

hence

$$
\xi \frac{\partial R}{\partial v}-R \frac{\partial \xi}{\partial z}=0
$$

$$
R=U \xi
$$

where $U$ is a function of $u$ alone. Using (3) we may write the second equation of (2) in the form

(4) $U \xi\left(p r_{1}-p_{1} r\right)-q_{1}\left(\xi+\xi U^{\prime}+U \frac{\partial \xi}{\partial u}\right)-q U \eta_{1} r=0$.

We shall therefore replace equations (2) by (3) and (4).

2. The Inversion of $C$. If we invert the circles $C$ relative to the circles $x^{2}+z^{2}=K^{2}, y=0$, where $K$ is any constant, we get the following system of lines $L$,

$$
x=\frac{K^{2}}{2 R}, \quad y=0,
$$

* Presented to the Society, March 25, 1932.

† See Eisenhart, Differential Geometry of Curves and Surfaces, Ex. 11, p. 444.

$\ddagger$ Eisenhart, p. 170 . 
which will constitute a rectilinear congruence. Let us determine the condition that this congruence be normal.

Any point on the lines (5) will have the coordinates $\left(K^{2} /(2 R)\right.$, $0, z)$. The necessary and sufficient condition that (5) shall define a normal congruence is that $\delta z=0$ for all values of $d v / d u$. Hence

or

$$
\frac{\partial z}{\partial u} d u+\frac{\partial z}{\partial v} d v-\frac{K^{2}}{2 R}\left(q d u+q_{1} d v\right) \equiv 0,
$$

$$
\frac{\partial z}{\partial u}=\frac{K^{2} q}{2 R}, \quad \frac{\partial z}{\partial v}=\frac{K^{2} q_{1}}{2 R} .
$$

The condition of integrability is that $\partial^{2} z / \partial u \partial v=\partial^{2} z / \partial v \partial u$; hence

$$
R \frac{\partial q}{\partial v}-q \frac{\partial R}{\partial v}=R \frac{\partial q_{1}}{\partial u}-q_{1} \frac{\partial R}{\partial u}
$$

which by means of (3) becomes*

$$
U \xi\left(r p_{1}-r_{1} p\right)+q U \eta_{1} r+q_{1}\left(\xi U^{\prime}+U \frac{\partial \xi}{\partial u}\right)=0 .
$$

On making use of (4), this further reduces to $q_{1}=0$; hence we have the following theorem.

TheOREM 1. A necessary and sufficient condition that the lines $L$, obtained by the above inversion of the cyclic system $C$, shall form a normal congruence, is that $S$ be referred to its lines of curvature.

We readily find that for $q_{1}=p=0$, (4) becomes $r\left(\xi p_{1}+\eta_{1} q\right)=0$. Two cases are to be considered.

(a) If $r=0$, the curves $v=$ const. are geodesics; and since they are lines of curvature, they are plane curves. Consequently the planes of $C$ constitute but a one-parameter family, and their envelope is the developable surface which is readily seen to be one of the focal sheets of $S$. We note also from (2) that for $r=0, R$ is a function of $u$ alone.

(b) If $\xi p_{1}+\eta_{1} q=0$, it is readily seen $\dagger$ that $D: E=D^{\prime \prime}: G$. Hence $S$ is in this case either a sphere or a plane. If $S$ is a sphere the planes of $C$ pass through the center.t

* Eisenhart, p. 168.

$\dagger$ Eisenhart, p. 174.

$\ddagger$ Eisenhart, p. 441. 
3. The Focal Points of $C$. The direction cosines of an arbitrary tangent of $C$ are $(-\sin \theta, 0, \cos \theta)$. Since the displacements $\delta x, \delta y, \delta z$ of the focal points of $C$ must be in the direction of the tangents we have $\cos \theta \delta x+\sin \theta \delta z=0, \delta y=0$. On using (1), these equations may be written

$$
\begin{gathered}
\frac{x}{R} \frac{\partial R}{\partial u} d u+\frac{x}{R} \frac{\partial R}{\partial v} d v+\frac{\xi(x-R)}{R} d u-z\left(q d u+q_{1} d v\right)=0 \\
\eta_{1} d v+x\left(r d u+r_{1} d v\right)-z\left(p d u+p_{1} d v\right)=0
\end{gathered}
$$

Obviously, we must have

$$
\left|\begin{array}{cc}
\frac{x}{R} \frac{\partial R}{\partial u}+\frac{\xi(x-R)}{R}-q z & \frac{x}{R} \frac{\partial R}{\partial v}-q_{1} z \\
r x-p z & \eta_{1}+r_{1} x-p_{1} z
\end{array}\right|=0 .
$$

Hence the focal points of $C$, which are at most four in number, are given by (1) and (7).

Let us now consider the case when the inversion of $C$ leads to a normal rectilinear congruence. We have seen that we must have $p=q_{1}=0$, and $r\left(\xi p_{1}+\eta_{1} q\right)=0$. The first case, (a), is of some interest; for $p=q_{1}=r=0$, equation (7) becomes*

$$
\left(\frac{\partial R}{\partial u}+\xi\right) x-R q z-R \xi=0, \quad r_{1} x-p_{1} z+\eta_{1}=0 .
$$

We note that the two centers of principal curvature, $(0,0,-\xi / q)$ and $\left(0,0, \eta_{1} / p_{1}\right)$, lie in the planes $(8)$, while the second plane of (8) also contains the center of geodesic curvature, $\left(-\eta_{1} / r_{1}, 0,0\right)$, of the curve $u=$ const. Hence we have the following theorem.

THEOREM 2. If the above inversion of $C$ leads to a normal rectilinear congruence, and the curves $v=$ const. are geodesics, $(r=0)$, then two of the focal points of $C$ are collinear with the center of principal curvature of the curve $v=$ const., and the other two are collinear with the centers of principal and geodesic curvature of the curve $u=$ const.

From (6) it is evident that for $p=q_{1}=r=0$, and $R=f(u), d u$ and $d v$ are factors of these equations. Hence we have the following theorem.

* $R$ is a function for $u$ alone for (a). 
THEOREM 3. If the above inversion of $C$ leads to a normal rectilinear congruence, and the curves $v=$ const. are geodesics, then the circles of $C$ which have an envelope are those which correspond to the lines of curvature on $S$.

4. The Focal Points of $L$. The Developables of $L$. As the vertex of $T$ is displaced along the curves of $S$ which define the developables of $L$, the displacements $\delta x$ and $\delta y$ of the focal points will be zero. Hence from (5), when $L$ is a normal congruence, we have

$$
\begin{gathered}
\frac{K^{2}}{2 R^{2}}\left(\frac{\partial R}{\partial u} d u+\frac{\partial R}{\partial v} d v\right)-\xi d u-z q d u=0 \\
\eta_{1} d v+\frac{K^{2}}{2 R}\left(r d u+r_{1} d v\right)-z p_{1} d v=0 .
\end{gathered}
$$

The elimination of $d v / d u$ between equations (9) gives us a quadratic in $z$ whose roots determine the focal points of $L$; the elimination of $z$ gives the equation of the curves on $S$ defining the developables of $L$.

For (a), that is for $r=0, R=f(u)$, the focal points of $L$ as determined by (9) are

$$
z_{1}=\frac{1}{q}\left(\frac{K^{2}}{2 R^{2}} \frac{\partial R}{\partial u}-\xi\right), \quad z_{2}=\frac{1}{p_{1}}\left(\frac{K^{2} r_{1}}{2 R}+\eta_{1}\right) .
$$

When we put (5) in the second of equations (8) and solve for $z$ we get the value of $z_{2}$ in (10). Hence one of the focal points of $L$ is collinear with the two focal points of $C$ determined by the second member of (8). It is readily shown from (5), (8), and (10) that the other focal point of $L$ is not collinear with the other two focal points of $C$.

From (9) it is readily seen that for (a), $d u$ and $d v$ are factors of these equations. Hence we have the following theorem.

THEOREM 4. If the above inversion of $C$ leads to a normal rectilinear congruence $L$, and the curves $v=$ const. are geodesics, the lines of curvature on $S$ define both the developables of $L$, and those families of $C$ which have an envelope.

Wesleyan University 